\title{
Supplementary materials for: Biomass burning contribution to Beijing aerosol
}

Yuan Cheng ${ }^{1, *}$, Guenter Engling ${ }^{2}$, Ke-bin $\mathrm{He}^{1}$, Feng-kui Duan ${ }^{1}$, Yong-liang Ma ${ }^{1}$, Zhen-yu $\mathrm{Du}^{1}$, Jiu-meng Liu ${ }^{3}$, Mei, Zheng ${ }^{4}$, Rodney J. Weber ${ }^{3}$

1 State Key Joint Laboratory of Environment Simulation and Pollution Control, School of Environment, Tsinghua University, Beijing, China

2 Department of Biomedical Engineering and Environmental Sciences, National Tsing Hua University, Hsinchu, Taiwan

3 School of Earth and Atmospheric Sciences, Georgia Institute of Technology, Atlanta, Georgia, United States

4 College of Environmental Sciences and Engineering, Peking University, Beijing, China

* Corresponding author.

Tel: 86-10-62794331; Fax: 86-10-62771101

Email: ycheng@mail.tsinghua.edu.cn (chengyuan02@gmail.com)

Section 1. Summary of the published levoglucosan to $\mathrm{K}^{+}$and levoglucosan to mannosan ratios.

Section 2. Estimation of the uncertainties of OC, EC, WSOC, and water soluble ions.

Section 3. Estimating the contributions of the six factors resolved by PMF to the constructed $\mathrm{PM}_{2.5}$ mass using the multivariate linear regression.

Section 4. Statistical results for the concentrations, ratios, regressions and comparisons included in the present study. 
Section 1. Summary of the published levoglucosan to $\mathrm{K}^{+}$ratios $(\mathrm{L} / \mathrm{K})$ and the levoglucosan to mannosan ratios (L/M).

\begin{tabular}{|c|c|c|c|c|c|c|c|}
\hline Biomass type & Combustion style & levoglucosan & $\mathrm{K}^{+}$ & mannosan & $\mathrm{L} / \mathrm{K}$ & $\mathrm{L} / \mathrm{M}$ & Reference \\
\hline \multicolumn{8}{|l|}{ US hard wood } \\
\hline Red maple & fireplace & 9.28 & 1.24 & 0.28 & 7.51 & 33.02 & Fine et al., $2001^{\mathrm{a}}$ \\
\hline Northern red oak & fireplace & 14.72 & 1.00 & 0.42 & 14.71 & 35.46 & Fine et al., 2001 ${ }^{\mathrm{a}}$ \\
\hline Paper birch & fireplace & 9.51 & 0.98 & 0.11 & 9.74 & 83.43 & Fine et al., $2001^{\mathrm{a}}$ \\
\hline Yellow poplar & fireplace & 13.26 & 0.73 & 1.24 & 18.27 & 10.67 & Fine et al., $2002^{\mathrm{a}}$ \\
\hline White ash & fireplace & 7.60 & 1.75 & 0.59 & 4.34 & 12.88 & Fine et al., $2002^{\mathrm{a}}$ \\
\hline Sweetgum & fireplace & 10.09 & 0.80 & 0.55 & 12.65 & 18.38 & Fine et al., $2002^{\mathrm{a}}$ \\
\hline Mockernut hickory & fireplace & 11.81 & 0.20 & 0.48 & 59.06 & 24.73 & Fine et al., $2002^{\mathrm{a}}$ \\
\hline White oak & fireplace & 7.41 & 0.90 & 0.57 & 8.20 & 12.89 & Fine et al., $2004 \mathrm{a}^{\mathrm{a}}$ \\
\hline Sugar maple & fireplace & 17.37 & 0.66 & 0.88 & 26.44 & 19.76 & Fine et al., $2004 \mathrm{a}^{\mathrm{a}}$ \\
\hline Black oak & fireplace & 17.78 & 1.03 & 0.76 & 17.30 & 23.40 & Fine et al., 2004a \\
\hline American beech & fireplace & 5.62 & 0.40 & 0.33 & 14.04 & 16.89 & Fine et al., $2004 \mathrm{a}^{\mathrm{a}}$ \\
\hline Black cherry & fireplace & 22.65 & 1.90 & 1.15 & 11.91 & 19.65 & Fine et al., $2004 \mathrm{a}^{\mathrm{a}}$ \\
\hline Quaking aspen & fireplace & 12.54 & 0.45 & 0.87 & 28.12 & 14.46 & Fine et al., $2004 \mathrm{a}^{\mathrm{a}}$ \\
\hline Red maple & stove & 12.66 & 1.68 & 0.66 & 7.54 & 19.27 & Fine et al., 2004b ${ }^{a}$ \\
\hline White oak & stove & 6.93 & 1.51 & 0.31 & 4.58 & 22.70 & Fine et al., 2004b $\mathrm{b}^{\mathrm{a}}$ \\
\hline White oak (catalyst) & stove & 5.93 & 2.74 & 0.23 & 2.16 & 26.05 & Fine et al., $2004 b^{a}$ \\
\hline Sugar maple & stove & 10.76 & 1.01 & 0.66 & 10.61 & 16.31 & Fine et al., 2004b ${ }^{\mathrm{a}}$ \\
\hline Oak & open chamber & 500.00 & & 18.00 & & 27.78 & Engling et al., $2006^{\mathrm{b}}$ \\
\hline Oak & open chamber & 252.00 & & 7.80 & & 32.31 & Engling et al., $2006^{\mathrm{b}}$ \\
\hline Cottonwood & open chamber & 276.00 & & 20.00 & & 13.80 & Engling et al., $2006^{\mathrm{b}}$ \\
\hline Oak & fireplace & 0.71 & 0.03 & & 21.40 & & Schauer et al., $2001^{\circ}$ \\
\hline Eucalyptus & fireplace & 1.94 & 0.07 & & 28.21 & & Schauer et al., $2001^{\circ}$ \\
\hline
\end{tabular}




\begin{tabular}{|c|c|c|c|c|c|c|c|}
\hline \multicolumn{8}{|l|}{ US softwood } \\
\hline Eastern white pine & fireplace & 3.84 & 0.44 & 0.66 & 8.75 & 5.81 & Fine et al., $2001^{\mathrm{a}}$ \\
\hline Balsam fir & fireplace & 8.66 & 1.48 & 1.85 & 5.85 & 4.68 & Fine et al., $2001^{\mathrm{a}}$ \\
\hline Slash pine & fireplace & 4.72 & 0.65 & 0.96 & 7.23 & 4.91 & Fine et al., $2002^{\mathrm{a}}$ \\
\hline White spruce & fireplace & 12.72 & 0.42 & 3.23 & 30.22 & 3.94 & Fine et al., $2004 \mathrm{a}^{\mathrm{a}}$ \\
\hline Douglar fir & fireplace & 26.15 & 0.37 & 5.89 & 71.45 & 4.44 & Fine et al., $2004 \mathrm{a}^{\mathrm{a}}$ \\
\hline Pinyon pine & fireplace & 0.80 & 0.16 & 0.12 & 5.01 & 6.67 & Fine et al., $2004 \mathrm{a}^{\mathrm{a}}$ \\
\hline Pine & fireplace & 1.38 & 0.03 & & 52.25 & & Schauer et al., $2001^{\mathrm{c}}$ \\
\hline Loblolly pine & stove & 11.04 & 0.46 & 2.02 & 24.09 & 5.46 & Fine et al., $2004 b^{a}$ \\
\hline Douglas fir & stove & 31.80 & 1.17 & 9.15 & 27.16 & 3.47 & Fine et al., $2004 \mathrm{~b}^{\mathrm{a}}$ \\
\hline Douglas fir (catalyst) & stove & 25.67 & 0.62 & 4.41 & 41.34 & 5.82 & Fine et al., $2004 \mathrm{~b}^{\mathrm{a}}$ \\
\hline Ponderosa pine & open chamber & 80.00 & & 26.00 & & 3.08 & Engling et al., $2006^{\mathrm{b}}$ \\
\hline Western hemlock & open chamber & 3.18 & 0.69 & & 4.61 & & Hays et al., $2002^{\mathrm{a}}$ \\
\hline \multicolumn{8}{|l|}{ Europen hardwood } \\
\hline Beech & stove & 4.10 & 0.21 & 0.28 & 19.25 & 14.64 & Schmidl et al., $2008 \mathrm{a}^{\mathrm{a}}$ \\
\hline Oak & stove & 13.30 & 0.41 & 0.92 & 32.13 & 14.46 & Schmidl et al., $2008 \mathrm{a}^{\mathrm{a}}$ \\
\hline Eucalyptus & chamber & 14.70 & & 0.43 & & 34.19 & Gonçalves et al., $2010^{\mathrm{a}}$ \\
\hline Cork oak & chamber & 6.80 & & 0.27 & & 25.19 & Gonçalves et al., $2010^{\mathrm{a}}$ \\
\hline
\end{tabular}




\begin{tabular}{|c|c|c|c|c|c|c|c|}
\hline Golden wattle & chamber & 1.90 & & 0.17 & & 11.18 & Gonçalves et al., $2010^{\mathrm{a}}$ \\
\hline Beech & house & 2286.78 & & 155.02 & & 14.75 & Bari et al., 2009a \\
\hline \multicolumn{8}{|l|}{ Europen softwood } \\
\hline Spruce & stove & 10.70 & 0.16 & 3.00 & 66.88 & 3.57 & Schmidl et al., 2008a $\mathrm{a}^{\mathrm{a}}$ \\
\hline Larch & stove & 15.10 & 0.07 & 3.90 & 206.85 & 3.87 & Schmidl et al., 2008a ${ }^{a}$ \\
\hline Briquettes & stove & 10.10 & 0.19 & 4.00 & 53.44 & 2.53 & Schmidl et al., $2008 \mathrm{a}^{\mathrm{a}}$ \\
\hline Maritime pine & chamber & 3.80 & & 1.30 & & 2.92 & Gonçalves et al., $2010^{\mathrm{a}}$ \\
\hline Pine & chamber & 1.20 & 0.0046 & 0.32 & 260.87 & 3.75 & Iinuma et al., $2007^{\mathrm{c}}$ \\
\hline Pine with green needles & chamber & 0.85 & 0.084 & 0.13 & 10.12 & 6.54 & Iinuma et al., $2007^{\mathrm{c}}$ \\
\hline Spruce with green needles & chamber & 0.99 & 0.028 & 0.21 & 35.36 & 4.71 & Iinuma et al., $2007^{\mathrm{c}}$ \\
\hline \multicolumn{8}{|l|}{ US Needle } \\
\hline Needle & open chamber & 5.64 & 1.05 & 1.35 & 5.37 & 4.18 & Sullivan et al., $2008^{\mathrm{d}}$ \\
\hline Needle & open chamber & 25.53 & 4.66 & 8.32 & 5.48 & 3.07 & Sullivan et al., $2008^{\mathrm{d}}$ \\
\hline Needle & open chamber & 28.32 & 4.37 & 6.72 & 6.48 & 4.21 & Sullivan et al., $2008^{\mathrm{d}}$ \\
\hline Needle & open chamber & 4.76 & 0.70 & 1.64 & 6.80 & 2.90 & Sullivan et al., $2008^{\mathrm{d}}$ \\
\hline Needle & open chamber & 30.84 & 2.95 & 8.94 & 10.45 & 3.45 & Sullivan et al., $2008^{d}$ \\
\hline Needle & open chamber & 12.70 & 5.53 & 8.97 & 2.30 & 1.42 & Sullivan et al., $2008^{\mathrm{d}}$ \\
\hline Needle & open chamber & 25.01 & 7.19 & 14.25 & 3.48 & 1.76 & Sullivan et al., $2008^{\mathrm{d}}$ \\
\hline Needle & open chamber & 8.73 & 1.66 & 2.10 & 5.26 & 4.16 & Sullivan et al., $2008^{\mathrm{d}}$ \\
\hline Needle & open chamber & 10.53 & 1.07 & 6.42 & 9.84 & 1.64 & Sullivan et al., $2008^{\mathrm{d}}$ \\
\hline Needle & open chamber & 7.31 & 3.14 & 1.35 & 2.33 & 5.41 & Sullivan et al., $2008^{\mathrm{d}}$ \\
\hline Needle & open chamber & 186.00 & & 50.00 & & 3.72 & Engling et al., $2006^{\mathrm{b}}$ \\
\hline Needle & open chamber & 98.00 & & 15.00 & & 6.53 & Engling et al., $2006^{\mathrm{b}}$ \\
\hline Needle & open chamber & 530.00 & & 144.00 & & 3.68 & Engling et al., $2006^{\mathrm{b}}$ \\
\hline
\end{tabular}


Needle open chamber

US grass

Grass
Grass
Grass
Grass
Grass
Grass
Grass

open chamber

open chamber

open chamber

open chamber

open chamber

open chamber

open chamber

open chamber

open chamber

open chamber

open chamber

open chamber

open chamber

open chamber

open chamber

Duff

\section{Asia Rice straw}

Rice straw (Taiwan)

Rice straw (Taiwan)

Rice straw (Taiwan)

Rice straw (Taiwan)
247.00

66.00

3.74

Engling et al., 2006

$\begin{array}{cc}6.66 & 12.21 \\ 8.73 & 29.34 \\ 4.77 & 58.51 \\ 16.85 & 4.44 \\ 7.98 & 0.84 \\ 20.36 & 2.25 \\ 18.88 & 301.98\end{array}$

0.51

0.62

0.52

0.92

0.36

0.52

1.64

0.55

$0.30 \quad 14.08$

$0.08 \quad 9.17$

$3.80 \quad 18.32$

$9.50 \quad 22.17$

$9.05 \quad 39.15$

$0.06 \quad 11.51$

12.59

14.67

23.71

14.46

8.41

11.94

9.87

525.00

2.20

11.60

6.32

5.72

1.09

2.32

1.09

17.79

21.75

12.91

1.33

1.12

14.64

4.37

0.70

11.97

5.86

216.00

22.73

0.99

1.92

$17.06 \quad 1.00$

1.68

2.43

Sullivan et al., 2008 ${ }^{\mathrm{d}}$ Sullivan et al., $2008^{d}$ Sullivan et al., $2008^{\mathrm{d}}$ Sullivan et al., 2008 ${ }^{\mathrm{d}}$ Sullivan et al., $2008^{\mathrm{d}}$ Sullivan et al., $2008^{d}$ Sullivan et al., 2008 ${ }^{\mathrm{d}}$ Engling et al., 2006 ${ }^{\mathrm{c}}$

\begin{tabular}{lcccccc} 
open chamber & 1.97 & 6.84 & 0.16 & 0.29 & 12.31 & Sullivan et al., 2008 $^{\text {d }}$ \\
open chamber & 28.76 & 39.49 & 1.53 & 0.73 & 18.80 & Sullivan et al., 2008 $^{\text {d }}$ \\
open chamber & 33.02 & 36.29 & 0.60 & 0.91 & 55.03 & Sullivan et al., 2008 $^{\text {d }}$ \\
open chamber & 12.02 & 25.26 & 0.85 & 0.48 & 14.14 & Sullivan et al., 2008 $^{\text {d }}$ \\
\hline
\end{tabular}




\begin{tabular}{|c|c|c|c|c|c|c|c|}
\hline Rice straw (Taiwan) & open chamber & 25.69 & 21.09 & 0.76 & 1.22 & 33.80 & Sullivan et al., 2008 \\
\hline Rice straw (Taiwan) & open chamber & 25.05 & 38.58 & 0.81 & 0.65 & 30.93 & Sullivan et al., 2008 \\
\hline Rice straw (Taiwan) & open chamber & 7.44 & 25.66 & 0.15 & 0.29 & 49.60 & Sullivan et al., 2008 \\
\hline Rice straw (Bangladesh) & stove & 1.83 & 2.50 & 0.04 & 0.73 & 41.59 & Sheesley et al., 2003 \\
\hline Rice straw (Thailand) & open burning & 56.20 & 50.00 & & 1.12 & & Oanh et al., 2011 \\
\hline Rice straw (Taiwan) & filed burning & 0.932 & 3.908 & & 0.24 & & Engling et al., $2009^{\mathrm{d}}$ \\
\hline Rice straw (Taiwan) & filed burning & 1.67 & 3.167 & & 0.53 & & Engling et al., $2009^{\mathrm{d}}$ \\
\hline Rice straw (Taiwan) & filed burning & 2.527 & 4.452 & & 0.57 & & Engling et al., $2009^{\mathrm{d}}$ \\
\hline Rice straw (Taiwan) & filed burning & 1.200 & 4.172 & & 0.29 & & Engling et al., $2009^{\mathrm{d}}$ \\
\hline Rice straw (Taiwan) & filed burning & 3.1364 & & 0.123 & & 25.48 & Engling et al., $2009^{\mathrm{d}}$ \\
\hline
\end{tabular}

a wt $\%$ of PM mass

b $\mu \mathrm{g} / \mathrm{mg}-\mathrm{OC}$

c g/kg-fuel

$\mathrm{d} \mu \mathrm{g} / \mathrm{m}^{3}$ 
Section 2. Estimation of the uncertainties of OC, EC, WSOC, and water soluble ions.

Parallel analysis of OC, EC and water-soluble ions was not performed in the present study. However, their uncertainties could be estimated based on our previous work about the measurement method of ambient aerosol. Results from the inter-comparison of thermal-optical methods suggested that uncertainty of the total carbon (TC) quantified by the DRI carbon analyzer was about 3\% (Cheng et al., 2011, 2012a). On the other hand, the EC uncertainty could be calculated using the EC values measured by the bare and denuded quartz filters (the use of charcoal denuder will not influence the EC results, since EC exists only in the particle phase). Using this approach, the EC uncertainty was estimated to be about $4 \%$ based on the results from Cheng et al. (2010). Comparison of the TC uncertainty and the EC uncertainty indicates that the OC uncertainty should be comparable with EC. Therefore, a value of $5 \%$ was used as the uncertainty of $\mathrm{OC}$ and $\mathrm{EC}$ in the PMF model. Similarly, the uncertainty of $\mathrm{SO}_{4}{ }^{2-}$ and $\mathrm{K}^{+}$could also be calculated using their values measured by the bare and denuded quartz filters (the use of charcoal denuder will not influence the $\mathrm{SO}_{4}{ }^{2-}$ and $\mathrm{K}^{+}$results, since they exists only in the particle phase). Based on the results from Cheng et al. (2012b), the uncertainty of $\mathrm{SO}_{4}{ }^{2-}$ and $\mathrm{K}^{+}$was estimated to be about $3 \%$ and $4 \%$, respectively. In the PMF model, a value of $5 \%$ was used as the uncertainty of $\mathrm{SO}_{4}{ }^{2-}$ and $\mathrm{K}^{+}$. Oxalate was not measured by Cheng et al. (2012b), thus, it is assumed that the oxalate uncertainty was twice the value of that for $\mathrm{K}^{+}$(i.e., $10 \%$ ).

When performing the WSOC analysis at Georgia Institute of Technology, four ambient samples and eight sucrose solutions were analyzed twice. The results showed that the WSOC uncertainty was about $2 \%$ and $3 \%$ for the ambient samples and the sucrose solutions, respectively. In the PMF analysis, a value of 5\% was used as the WSOC uncertainty. 


\section{References}

Cheng Y., He K. B., Duan F. K., Zheng M., Ma Y. L., Tan J. H., and Du Z. Y.: Improved Measurement of Carbonaceous Aerosol: Evaluation of the sampling artifacts and inter-comparison of the thermal-optical analysis methods, Atmos. Chem. Phys., 10, $8533-8548,2010$.

Cheng Y., Duan F. K., He K. B., Zheng M., Du Z. Y., Ma Y. L., and Tan J. H.: Intercomparison of thermal-optical methods for the determination of organic and elemental carbon: Influences of aerosol composition and implications, Environ. Sci. Technol., 45, 10117-10123, 2011.

Cheng Y., Duan F. K., He K. B., Du Z. Y., Zheng M., and Ma Y. L.: Intercomparison of thermal-optical method with different temperature protocols: implications from source samples and solvent extraction, Atmos. Environ., 61, 453-462, 2012a.

Cheng Y., Duan F. K., He K. B., Du Z.Y ., Zheng M., and Ma Y. L.: Sampling artifacts of organic and inorganic aerosol: implications for the speciation measurement of particulate matter, Atmos. Environ., 55, 229-233, 2012 b. 
Section 3. Estimating the contributions of the six factors resolved by PMF to the constructed $\mathrm{PM}_{2.5}$ mass using the multivariate linear regression.

The contributions of the six factors resolved by PMF to the constructed $\mathrm{PM}_{2.5}$ mass were estimated using the multivariate linear regression:

$$
\begin{aligned}
{\left[\text { Constructed } P M_{2.5}\right] } & =K_{1} \times[\text { factor } 1]+K_{2} \times[\text { factor } 2]+K_{3} \times[\text { factor } 3]+K_{4} \times[\text { factor } 4] \\
& +K_{5} \times[\text { factor } 5]+K_{6} \times[\text { factor } 6]+b
\end{aligned}
$$

where [constructed $\mathrm{PM}_{2.5}$ ] is the constructed $\mathrm{PM}_{2.5}$ mass, [factor1] [factor6] is the factor contribution resolved by PMF, $\mathrm{K}_{1} \sim \mathrm{K}_{6}$ is the slope of each factor, and $\mathrm{b}$ is the constant. In this study, $\mathrm{R}^{2}$ of the multivariate linear regression was 0.99 . 
Section 4. Statistical results for the concentrations, ratios, regressions and comparisons included in the present study.

Table 4.1 Concentrations of $\mathrm{PM}_{2.5}$ components during summer and winter in Beijing. Values of OC, EC and WSOC are presented in $\mu \mathrm{gC} / \mathrm{m}^{3}$, whereas concentrations of the other species are presented in $\mu \mathrm{g} / \mathrm{m}^{3}$. Levoglucosan to $\mathrm{K}^{+}$and levoglucosan to mannosan ratios of the ambient samples are also shown.

\begin{tabular}{|c|c|c|c|c|c|c|c|c|c|c|c|c|c|c|c|c|}
\hline & $\mathrm{OC}$ & $\mathrm{EC}$ & WSOC & levo. & manno. & $\mathrm{K}^{+}$ & $\mathrm{NH}_{4}^{+}$ & $\mathrm{SO}_{4}^{2-}$ & $\mathrm{NO}_{3}^{-}$ & $\mathrm{C}_{2} \mathrm{O}_{4}{ }^{2-}$ & $\mathrm{Cl}^{-}$ & $\mathrm{Ca}^{2+}$ & $\mathrm{Na}^{+}$ & $\mathrm{Mg}^{2+}$ & levo. $/ \mathrm{K}^{+}$ & levo./manno. \\
\hline \multicolumn{17}{|c|}{ typical summer } \\
\hline minimum & 5.14 & 0.92 & 2.72 & 0.06 & 0.00 & 0.09 & 0.44 & 1.44 & 0.73 & 0.06 & 0.04 & 0.10 & n.a. & 0.01 & 0.05 & 7.44 \\
\hline lower quartile & 8.06 & 1.69 & 5.10 & 0.07 & 0.01 & 0.38 & 2.83 & 7.66 & 2.20 & 0.24 & 0.29 & 0.29 & 0.05 & 0.08 & 0.10 & 10.55 \\
\hline median & 9.32 & 2.02 & 5.88 & 0.10 & 0.01 & 0.68 & 9.77 & 19.00 & 13.72 & 0.36 & 0.80 & 0.45 & 0.13 & 0.11 & 0.16 & 12.50 \\
\hline upper quartile & 11.43 & 2.54 & 7.49 & 0.16 & 0.01 & 1.10 & 16.88 & 28.64 & 24.77 & 0.42 & 1.37 & 0.61 & 0.20 & 0.13 & 0.23 & 14.97 \\
\hline maximum & 18.71 & 4.21 & 11.95 & 0.26 & 0.02 & 2.60 & 42.79 & 70.89 & 69.74 & 0.90 & 6.00 & 0.80 & 0.39 & 0.22 & 0.69 & 23.05 \\
\hline \multicolumn{17}{|c|}{ BB summer } \\
\hline minimum & 7.62 & 1.76 & 5.05 & 0.10 & 0.01 & 2.33 & 7.30 & 23.65 & 8.56 & 0.39 & 0.94 & 0.24 & 0.16 & 0.07 & 0.04 & 12.97 \\
\hline lower quartile & 15.66 & 3.14 & 11.85 & 0.40 & 0.02 & 3.62 & 14.76 & 27.05 & 16.24 & 0.69 & 1.25 & 0.35 & 0.26 & 0.12 & 0.09 & 18.52 \\
\hline median & 20.78 & 3.95 & 14.58 & 0.46 & 0.02 & 5.70 & 15.82 & 40.55 & 23.72 & 0.85 & 1.78 & 0.39 & 0.32 & 0.14 & 0.10 & 21.65 \\
\hline upper quartile & 23.99 & 4.81 & 16.35 & 0.65 & 0.03 & 7.40 & 20.17 & 42.12 & 29.58 & 0.89 & 2.90 & 0.65 & 0.37 & 0.16 & 0.13 & 22.98 \\
\hline maximum & 38.12 & 6.15 & 25.98 & 2.30 & 0.12 & 10.10 & 27.57 & 44.92 & 46.30 & 1.41 & 11.27 & 0.83 & 0.62 & 0.21 & 0.24 & 56.72 \\
\hline \multicolumn{17}{|c|}{ typical winter } \\
\hline minimum & 5.50 & 1.77 & 1.98 & 0.06 & n.a. & 0.14 & 0.73 & 2.34 & 0.86 & 0.03 & 0.48 & 0.17 & 0.06 & 0.06 & 0.24 & 4.39 \\
\hline lower quartile & 15.43 & 4.83 & 7.00 & 0.33 & 0.05 & 0.80 & 3.41 & 5.86 & 4.89 & 0.08 & 3.90 & 1.19 & 0.48 & 0.13 & 0.40 & 8.43 \\
\hline median & 22.81 & 7.77 & 9.17 & 0.61 & 0.06 & 1.02 & 5.49 & 9.97 & 8.63 & 0.14 & 5.23 & 1.45 & 0.65 & 0.18 & 0.48 & 9.36 \\
\hline upper quartile & 29.10 & 9.44 & 14.72 & 0.88 & 0.10 & 1.56 & 10.54 & 15.95 & 14.47 & 0.18 & 6.82 & 2.30 & 0.81 & 0.22 & 0.62 & 9.96 \\
\hline maximum & 67.41 & 28.39 & 31.08 & 1.94 & 0.19 & 3.16 & 26.88 & 45.52 & 30.69 & 0.41 & 14.12 & 3.15 & 1.41 & 0.47 & 0.82 & 11.69 \\
\hline
\end{tabular}




\begin{tabular}{|c|c|c|c|c|c|c|c|c|c|c|c|c|c|c|c|c|}
\hline \multicolumn{17}{|c|}{ fireworks winter } \\
\hline minimum & 6.05 & 2.17 & 2.85 & 0.08 & 0.01 & 0.38 & 1.15 & 3.19 & 1.42 & 0.05 & 1.13 & 0.37 & 0.21 & 0.11 & 0.01 & 9.52 \\
\hline lower quartile & 12.40 & 3.62 & 6.16 & 0.29 & 0.02 & 2.23 & 2.42 & 8.47 & 3.37 & 0.10 & 3.49 & 0.66 & 0.43 & 0.25 & 0.10 & 9.95 \\
\hline median & 16.98 & 4.96 & 7.91 & 0.39 & 0.03 & 2.70 & 5.60 & 12.58 & 11.18 & 0.16 & 5.47 & 0.79 & 0.61 & 0.32 & 0.15 & 10.37 \\
\hline upper quartile & 38.24 & 7.82 & 19.04 & 0.64 & 0.06 & 5.06 & 16.74 & 31.32 & 27.50 & 0.23 & 10.28 & 1.20 & 1.04 & 0.44 & 0.21 & 11.32 \\
\hline maximum & 73.14 & 21.43 & 35.76 & 1.09 & 0.11 & 45.76 & 37.39 & 70.32 & 66.65 & 0.47 & 28.09 & 1.65 & 1.38 & 1.79 & 0.32 & 13.24 \\
\hline
\end{tabular}


Table 4.2 Statistical results of the linear regression analysis included in the present study.

\begin{tabular}{|c|c|c|c|c|c|c|c|}
\hline Y & $X$ & slope & $\begin{array}{c}\text { confidence } \\
\text { interval of slope }\end{array}$ & intercept & $\begin{array}{c}\text { confidence } \\
\text { interval of } \\
\text { intercept }\end{array}$ & $\mathrm{R}^{2}$ & Note \\
\hline levo. & $\mathrm{K}^{+}$ & $0.05 \pm 0.01$ & $0.03 \sim 0.08$ & $0.07 \pm 0.01$ & $0.05 \sim 0.10$ & 0.34 & $\begin{array}{l}\text { typical } \\
\text { summer }\end{array}$ \\
\hline levo. & $\mathrm{K}^{+}$ & $0.50 \pm 0.04$ & $0.43 \sim 0.57$ & $0.03 \pm 0.05$ & $-0.08 \sim 0.13$ & 0.82 & $\begin{array}{l}\text { typical } \\
\text { winter }\end{array}$ \\
\hline levo. & manno. & $9.93 \pm 0.97$ & $7.97 \sim 11.90$ & $0.02 \pm 0.01$ & $0.00 \sim 0.04$ & 0.73 & $\begin{array}{l}\text { typical } \\
\text { summer }\end{array}$ \\
\hline levo. & manno. & $17.67 \pm 1.61$ & $13.85 \sim 21.49$ & $0.14 \pm 0.08$ & $-0.05 \sim 0.33$ & 0.95 & $\begin{array}{c}\mathrm{BB} \\
\text { summer }\end{array}$ \\
\hline levo. & manno. & $9.41 \pm 0.22$ & $8.98 \sim 9.85$ & $0.01 \pm 0.02$ & $-0.02 \sim 0.05$ & 0.97 & $\begin{array}{l}\text { whole } \\
\text { winter }\end{array}$ \\
\hline
\end{tabular}


Table 4.3 Comparison of levoglucosan to $\mathrm{K}^{+}$ratios among different types of biomass: $\mathrm{p}$ values of the Independent-Samples $T$ Test $(p<0.1$ indicates significant difference at a 95\% level of confidence, whereas $\mathrm{p}>0.1$ indicates insignificant difference). The Independent-Samples $\mathrm{T}$ Test was not performed between EU hardwood and the other types of biomass because few data are available for EU hardwood.

\begin{tabular}{|c|c|c|c|c|c|c|c|c|}
\hline & $\begin{array}{c}\text { US } \\
\text { hardwood }\end{array}$ & $\begin{array}{c}\text { US } \\
\text { softwood }\end{array}$ & $\begin{array}{c}\text { EU } \\
\text { hardwood }\end{array}$ & $\begin{array}{c}\text { EU } \\
\text { softwood }\end{array}$ & $\begin{array}{c}\text { US } \\
\text { needles }\end{array}$ & $\begin{array}{c}\text { US } \\
\text { grass }\end{array}$ & $\begin{array}{l}\text { US } \\
\text { duff }\end{array}$ & $\begin{array}{c}\text { Asian } \\
\text { rice } \\
\text { straw }\end{array}$ \\
\hline $\begin{array}{c}\text { US } \\
\text { hardwood }\end{array}$ & & 0.306 & & 0.086 & 0.003 & 0.019 & 0.985 & 0.000 \\
\hline $\begin{array}{c}\text { US } \\
\text { softwood } \\
\text { EU }\end{array}$ & & & & 0.103 & 0.007 & 0.003 & 0.523 & 0.001 \\
\hline hardwood & & & & & & & & \\
\hline $\begin{array}{c}\text { EU } \\
\text { softwood }\end{array}$ & & & & & 0.063 & 0.059 & 0.086 & 0.054 \\
\hline $\begin{array}{c}\text { US } \\
\text { needles }\end{array}$ & & & & & & 0.171 & 0.028 & 0.000 \\
\hline US grass & & & & & & & 0.003 & 0.143 \\
\hline US duff & & & & & & & & 0.008 \\
\hline $\begin{array}{c}\text { Asian } \\
\text { rice straw }\end{array}$ & & & & & & & & \\
\hline
\end{tabular}


Table 4.4 Comparison of levoglucosan to mannosan ratios among different types of biomass: $p$ values of the Independent-Samples T Test $(p<0.1$ indicates significant difference at a $95 \%$ level of confidence, whereas $\mathrm{p}>0.1$ indicates insignificant difference).

\begin{tabular}{|c|c|c|c|c|c|c|c|c|}
\hline & $\begin{array}{c}\text { US } \\
\text { hardwood }\end{array}$ & $\begin{array}{c}\text { US } \\
\text { softwood }\end{array}$ & $\begin{array}{c}\text { EU } \\
\text { hardwood }\end{array}$ & $\begin{array}{c}\text { EU } \\
\text { softwood }\end{array}$ & $\begin{array}{c}\text { US } \\
\text { needles }\end{array}$ & $\begin{array}{c}\text { US } \\
\text { grass }\end{array}$ & $\begin{array}{l}\text { US } \\
\text { duff }\end{array}$ & $\begin{array}{l}\text { Asian } \\
\text { rice } \\
\text { straw }\end{array}$ \\
\hline $\begin{array}{c}\text { US } \\
\text { hardwood }\end{array}$ & & 0.000 & 0.455 & 0.002 & 0.000 & 0.357 & 0.000 & 0.264 \\
\hline $\begin{array}{c}\text { US } \\
\text { softwood }\end{array}$ & & & 0.009 & 0.448 & 0.082 & 0.012 & 0.000 & 0.001 \\
\hline $\begin{array}{c}\text { EU } \\
\text { hardwood }\end{array}$ & & & & 0.008 & 0.007 & 0.875 & 0.005 & 0.101 \\
\hline $\begin{array}{c}\text { EU } \\
\text { softwood }\end{array}$ & & & & & 0.517 & 0.010 & 0.000 & 0.001 \\
\hline $\begin{array}{c}\text { US } \\
\text { needles }\end{array}$ & & & & & & 0.009 & 0.001 & 0.001 \\
\hline US grass & & & & & & & 0.005 & 0.071 \\
\hline US duff & & & & & & & & 0.000 \\
\hline $\begin{array}{c}\text { Asian } \\
\text { rice straw }\end{array}$ & & & & & & & & \\
\hline
\end{tabular}

